\title{
Elucidation of Seam Tracking Based on Keyhole Characteristic during High-Power Fiber Laser Welding
}

\author{
Yonghua Liu ${ }^{a}$, Xiangdong Gao ${ }^{b, *}$ and Deyong You \\ School of Electromechanical Engineering, Guangdong University of Technology, \\ No.100 West Waihuan Road, Higher Education Mega Center, Panyu District, Guangzhou 510006, \\ China \\ ayh880819@126.com, bgaoxd666@126.com *Corresponding author
}

Keywords: High-power fiber laser welding; Keyhole; Seam tracking

\begin{abstract}
During deep penetration laser welding, a deep cavity called a keyhole is formed in a molten pool due to the intense recoil pressure of evaporation. The welding quality and stability are related to the configuration and characteristics of a keyhole. Some characteristic parameters of a keyhole can be extracted as an important method for seam tracking. The fiber laser butt-joint welding of Type 304 austenitic stainless steel plate with a high power $10 \mathrm{~kW}$ continuous wave fiber laser was studied and an infrared sensitive high-speed video camera was used to capture the dynamic images of the molten pools. In this paper the centroid point horizontal coordinate and column coordinate of a keyhole were defined as the keyhole characteristic parameters. By analyzing the change of the keyhole characteristic parameters during welding, it was found that these parameters could reflect the condition of seam tracking and the stability of laser welding as well as the quality of laser welding effectively, and then the status of welding could be monitored and distinguished by characteristic of a keyhole during high-power fiber laser welding.
\end{abstract}

\section{Introduction}

Compared with the traditional welding methods, the high-power fiber laser welding has become an important welding technology due to quicker welding speed, bigger depth-to-width ratio and smaller heat affected zone [1,2]. Because of many disturbances in welding process, the unstable state still appears during high-power fiber laser welding. Various kinds of errors of the system such as welding fixture assembly error, thermal deformation during the welding and so on directly affect the quality and stability of welding and bring about difficulty for seam tracking [3]. Therefore, the welding process should be real-time tracked to realize welding automatic control. Visual sensing method is often applied to monitor the laser welding process [4]. In seam tracking based on vision sensors, welding image processing is the most basic and the most key link, and the quality of welding image processing such as extracting characteristic parameters of a keyhole directly affect the seam tracking [5]. The centroid point horizontal coordinate and column coordinate of a keyhole were defined as the keyhole characteristic parameters. The relation between change of characteristic parameters of a keyhole and the seam tracking and welding stability was explored. According to these keyhole parameters, the seam tracking could be monitored, and it is valuable to control the welding quality and evaluate the welding stability.

\section{Experimental Setup}

The experimental system comprised an IPG YLR-10000 fiber laser welding device (laser power $10 \mathrm{~kW}$ ), a Panasonic 6-axis robot, a welding experimental platform equipped with servo motors and with a high speed camera, a set of image acquisition system and monitor, and shielding gas (argon). The schematic drawing of the whole experimental system is shown in Fig. 1. In order to extract near infrared images of molten pools and eliminate the interference, a combination filtering system with a filter length of 960-990nm was placed in front of the vision sensor. A fiber laser butt-joint welding of 
Type 304 austenitic stainless steel plate with a high power $10 \mathrm{~kW}$ continuous wave fiber laser was designed, and an infrared sensitive high-speed video camera was used to capture the dynamic images of the molten pools which were carried out at 1000 frames/s. The size of each image was $512 \times 512$ pixel. The welding path was an oblique trajectory, which reflected the actual situation where the deviation was left or right from the weld seam centre [6]. The material used was a stainless steel plate with dimensional size of $150 \times 100 \times 10 \mathrm{~mm}$, and the seam gap was less than $0.1 \mathrm{~mm}$. The welding speed was $2.5 \mathrm{~m} / \mathrm{min}$.

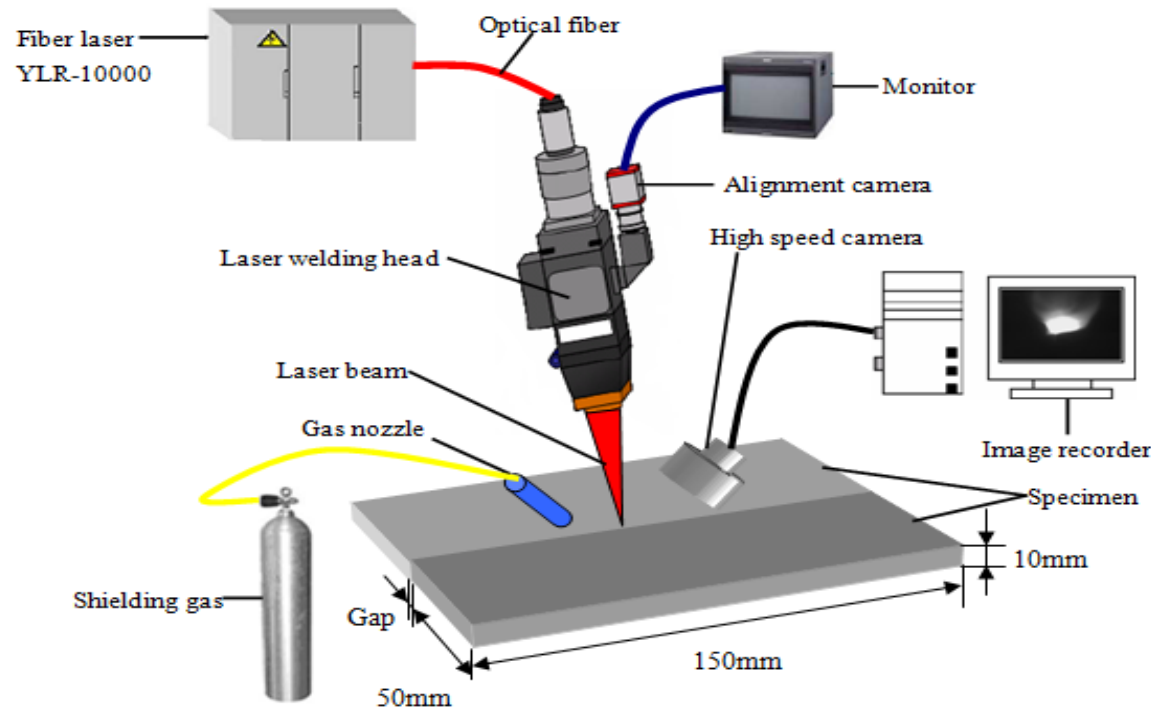

Fig. 1 Schematic of fiber laser welding experimental setup

\section{Welding Experiments and Discussion}

Image Preprocessing. An original and other processed keyhole images are shown in Fig.2. The original image (Fig.2a) was a grayscale image converted from RGB image and the median filtering was used to eliminate the noise jamming (Fig.2b). Gray stretch processing made the image dynamic range expanding and facilitated subsequent processing (Fig.2c). Then the gray threshold segmentation was used to segment the gray stretch image. As the brightness of the keyhole was much higher than elsewhere, here segmentation threshold of 0.9 was used, which could accurately extract the keyhole and reflect the configuration of the keyhole. Fig.2d shows a segmented image. Finally the corrosion operation and inflation operation were used to make the image contour smoothing, disconnect the narrow connection and eliminate fine protrusions, which could effectively give preparation for the subsequent feature extraction. A corrosion operation image and inflation operation image are shown in Fig. 2e and in Fig. 2f, respectively.

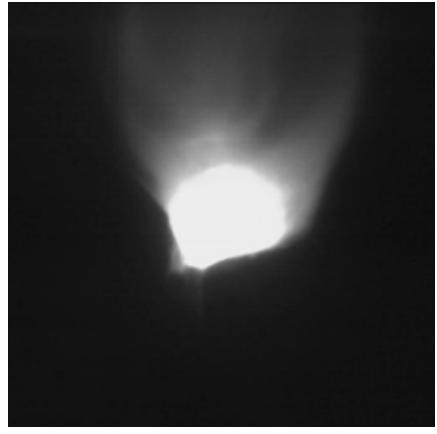

(a) Original image

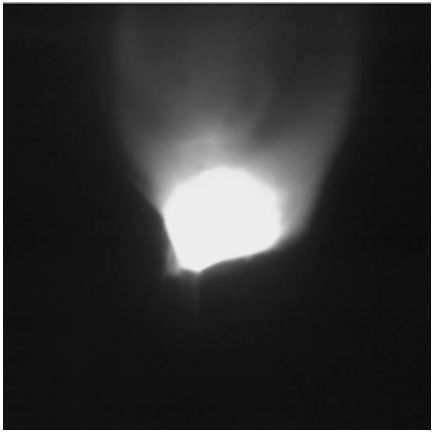

(b) Median filtering image

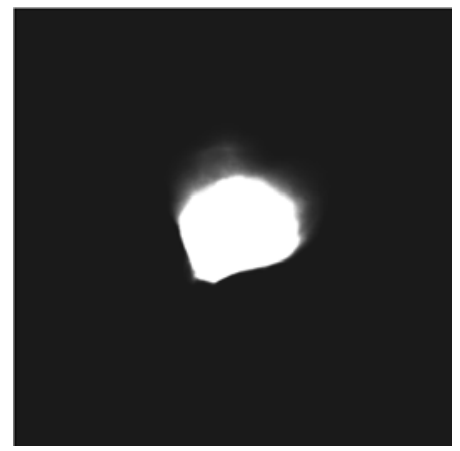

(c) Gray stretch image 


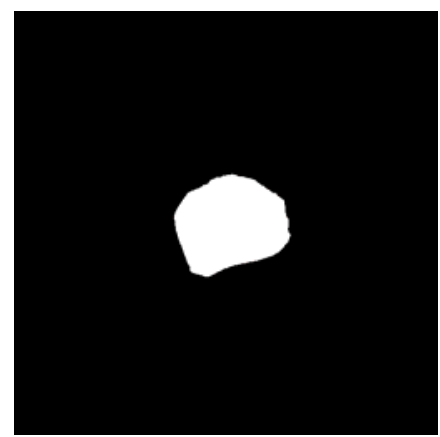

(d) Segmented image

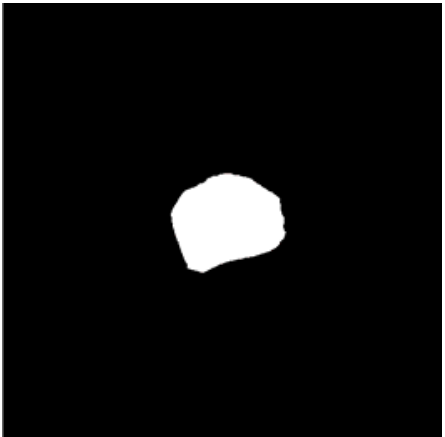

(e) Corrosion operation image

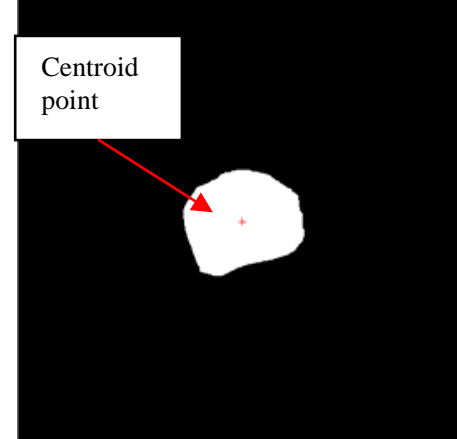

(f) Inflation operation image

Fig.2 Keyhole images of high-power fiber laser welding

Characteristic Parameters of Keyhole. One of characteristic parameters of a keyhole is the centroid point. The centroid point of a keyhole (Fig. 2f) can be obtained from the following formulas.

$$
C_{-} x=\frac{\sum_{j}^{n} \sum_{i}^{m} f(i, j)^{*} j}{\sum_{i}^{m} \sum_{j}^{n} f(i, j)} \quad C_{-} y=\frac{\sum_{i}^{m} \sum_{j}^{n} f(i, j) * i}{\sum_{i}^{m} \sum_{j}^{n} f(i, j)}
$$

where $C_{-} x$ is the value of centroid point horizontal coordinate, $C_{-} y$ is the value of column coordinate. $i$ and $j$ represent the column coordinate and horizontal coordinate of segmented image, respectively. $m$ and $n$ represent the number of horizontal and column, respectively, and $f(i, j)$ is the segmented image. Through calculation of the keyhole, the values of centroid point horizontal coordinate and column coordinate of the keyhole were respectively 262 (pixel) and 258 (pixel).

Analysis of Experimental Results. Fig.3 shows the actual welding workpiece. The measuring values of centroid point of keyholes with image sequences are shown in Fig. 4. Totally 2811 molten pool images were chosen to analyze the change of keyholes. During the welding, the starting point was $0.83 \mathrm{~mm}$ left from the weld center and the termination point was $0.76 \mathrm{~mm}$ right from the weld center. It was found that there existed apparent relationship between the weld deviation and the centroid column coordinate of keyholes.

The horizontal coordinate of centroid point is shown in Fig. 4a. The column coordinate of centroid point is shown in Fig. 4b. It was not found that there existed apparent relationship between the weld deviations and the horizontal coordinate of centroid point. The image sequences from 100 to 400 showed that the column coordinate of centroid point remained relatively unchanged, since the laser beam was far left from the weld center and didn't contact the weld center, and the temperature of workpiece was relatively low. The image sequences from 401 to 1500 showed that the column coordinate of centroid point gradually increased, which reflected the process that the laser beam was from just contacting with the weld seam to the just weld center. The section of image sequences from 1501 to 2000 showed that the column coordinate of centroid point gradually reduced, Since at this section the laser beam was from the just weld center to the right edge of weld centre. Then laser beam began to deviate right from the weld, as shown in the image sequences from 2001 to 2200. The section of image sequences from 2201 to 2910 showed that the column coordinate of centroid point unexpectedly gradually increased though the laser beam completely deviated right from the weld centre, the reason was that under the effect of the heat transfer, the temperature of the whole workpiece was very high at this section and then the welding process reached a new stable state.

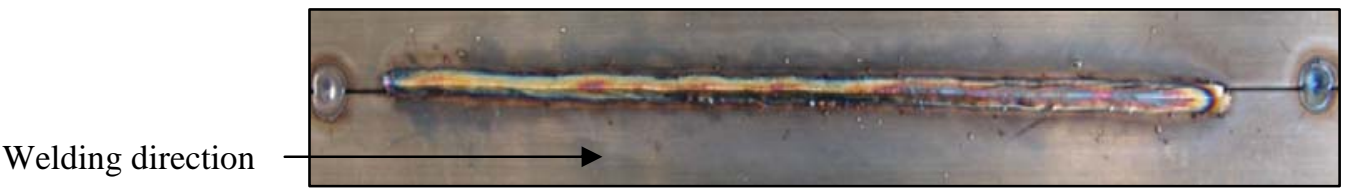

Fig. 3 Bead top of welded workpiece

Based on above analysis, it was found that the keyhole shape parameters of centroid point was an important parameter for seam tracking, that the change of the column coordinate of centroid of 
keyhole could effectively reflect the actual welding path, and the characteristic parameters could be used to detect the welding seam deviations.

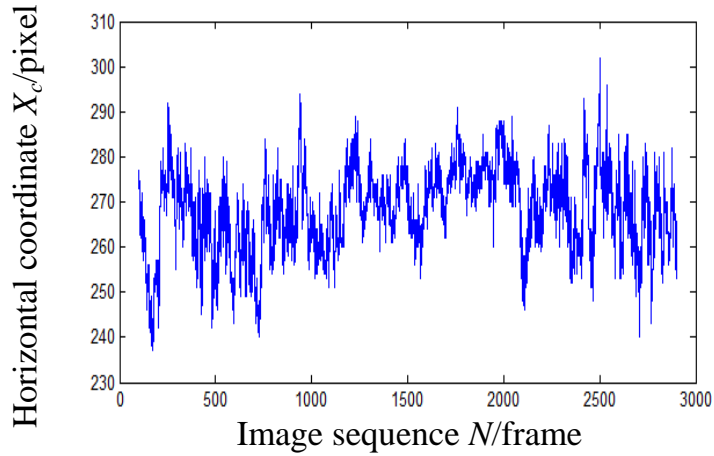

(a) Horizontal coordinate of centroid point

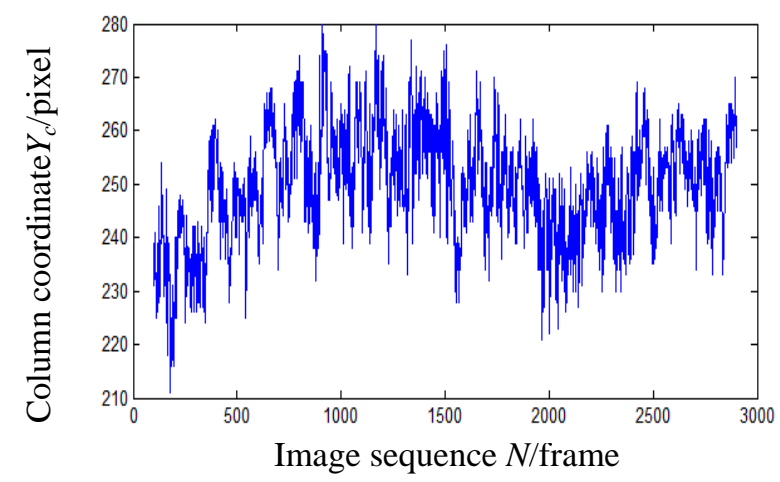

(b) Column coordinate of centroid point

Fig. 4 Keyhole shape parameters of centroid point

\section{Conclusions}

Image preprocessing method such as median filtering, gray stretch, threshold segmentation, corrosion operation and inflation operation can be applied to obtain the characteristic parameters of the keyhole.

The characteristic parameters of the keyhole such as column coordinate of centroid point can reflect the state of high-power fiber laser welding process and the condition of seam tracking.

Based on the characteristic parameters of the keyhole and the condition of seam tracking, the quality and stability of welding can be monitored.

\section{Acknowledgements}

This work was partly supported by the Specialized Research Fund for the Doctoral Program of Higher Education of China (20104420110001), the National Natural Science Foundation of China (51175095) and the Natural Science Foundation of Guangdong Province of China (10251009001000001, 9151009001000020). Many thanks are given to Katayama Laboratory of Osaka University, for their assistance of laser welding experiments.

\section{References}

[1] L.F. Mei, G.Y. Chen, X.Z. Jin, Y. Zhang and Q. Wu: Optics and Laser in Engineering, Vol. 47-11 (2009), p. 1117

[2] R.M. Miranda, G. Lopes , L. Quintino, J.P. Rodrigues and S. Williams: Materials \& Design, Vol. 29-10 (2008), p. 2072

[3] G.H. Li, C. Yan and Y.X. Wu: Optics and Lasers in Engineering, Vol. 47-9 (2009), p. 990

[4] X.B. Qi :Transactions of the China Welding Institution, Vol. 29-2 (2008), p. 108

[5] J.Q. Shen, S.S. Hu and S.Q. Feng: Journal of Tianjin University, Vol. 44-4(2011), p. 308

[6] X.D. Gao, D.Y. You and S.J. Katayama: Transactions of the China Welding Institution, Vol. 32-5 (2011), p. 49 